\title{
Co-ingestion of whole eggs or egg whites with glucose protects against postprandial hyperglycaemia-induced oxidative stress and dysregulated arginine metabolism in association with improved vascular endothelial function in prediabetic men
}

\author{
Joshua D. McDonald ${ }^{1}$, Eunice Mah ${ }^{1,2}$, Chureeporn Chitchumroonchokchai ${ }^{1}$, Elizabeth J. Reverri ${ }^{1}$, \\ Jinhui $\mathrm{Li}^{1}$, Jeff S. Volek ${ }^{3}$, Frederick A. Villamena ${ }^{4}$ and Richard S. Bruno ${ }^{1 *}$ \\ ${ }^{1}$ Human Nutrition Program, Department of Human Sciences, The Obio State University, Columbus, OH 43210, USA \\ ${ }^{2}$ Biofortis Inc., Addison, IL 60101, USA \\ ${ }^{3}$ Kinesiology Program, Department of Human Sciences, The Obio State University, Columbus, OH 43210, USA \\ ${ }^{4}$ Department of Biological Chemistry and Pharmacology, The Obio State University, Columbus, OH 43210, USA \\ (Submitted 28 March 2018 - Final revision received 21 May 2018 - Accepted 17July 2018 - First published online 30 August 2018)
}

\section{Abstract}

Replacing a portion of a glucose challenge with whole eggs (EGG) or egg whites (WHITE) was shown to protect against glucose-induced impairments in vascular function. We hypothesised in the present study that previously observed vasoprotection following co-ingestion of EGG or WHITE with glucose was attributed to limiting postprandial hyperglycaemia-induced oxidative stress that improves NO bioavailability. Prediabetic men completed a randomised, cross-over study in which they ingested isoenergetic meals containing $100 \mathrm{~g}$ glucose (GLU), or 75 g glucose with $1.5 \mathrm{EGG}$, seven WHITE or two egg yolks (YOLK). At 30 min intervals for $3 \mathrm{~h}$, we assessed plasma NO metabolites, the lipid peroxidation biomarker malondialdehyde, antioxidants, arginine and its methylated metabolites (asymmetric dimethylarginine and symmetric dimethylarginine), tetrahydrobiopterin redox status, vasoconstrictors and inflammatory markers. Compared with GLU, malondialdehyde was lower and NO ${ }^{\circ}$ metabolites were greater in EGG and WHITE, but YOLK was not different from GLU. Malondialdehyde was inversely correlated with $\mathrm{NO}^{\bullet}$ metabolites and vascular function, whereas $\mathrm{NO}^{\bullet}$ metabolites were positively correlated with vascular function. Compared with GLU, arginine was greater, but asymmetric and symmetric dimethylarginine and angiotensin-II were lower in all eggbased meals. Antioxidants, tetrahydrobiopterin redox status and inflammatory markers did not differ among treatments. Thus, while each eggbased meal improved arginine metabolism, only EGG and WHITE limited lipid peroxidation. This suggests that vasoprotection mediated by EGG and WHITE likely occurs in an $\mathrm{NO}^{\circ}$-dependent manner by improving arginine metabolism and attenuating oxidative stress that otherwise limit $\mathrm{NO}^{\bullet}$ biosynthesis and bioavailability to the vascular endothelium.

Key words: Oral glucose challenge: Flow-mediated dilation: Oxidative stress: Nitric oxide

CVD continues to be the leading cause of mortality in the $\mathrm{USA}^{(1)}$. Although CVD risk is attributable to numerous factors, findings from a meta-analysis indicate that the magnitude of postprandial hyperglycaemia (PPH) induces endothelial dysfunction $^{(2)}$. Independently, endothelial dysfunction precedes atherosclerosis ${ }^{(3)}$, supporting that managing $\mathrm{PPH}$ would protect against CVD-related morbidity. In agreement, controlled studies demonstrate that PPH impairs vascular endothelial function (VEF) in an oxidative stress-dependent manner likely by decreasing $\mathrm{NO}^{\bullet}$ bioavailability ${ }^{(4)}$. VEF is primarily regulated by $\mathrm{NO}^{\bullet}$ and its diminished bioavailability increases CVD risk $^{(5)}$. PPH-mediated oxidative stress is centrally implicated in impairing $\mathrm{VEF}^{(6)}$. Indeed, lipid peroxidation increases immediately following an oral glucose tolerance test (OGTT) in healthy adults $^{(4)}$. Increases in lipid peroxidation are also associated with decreases in brachial artery flow-mediated dilation (FMD), a functional index of $\mathrm{NO}^{\circ}$-dependent $\mathrm{VEF}^{(7)}$ that is prognostic of future CVD-related mortality ${ }^{(8)}$. Thus, dietary modifications that

Abbreviations: ADMA, asymmetric dimethylarginine; Ang-II, angiotensin-II; ARG, l-arginine; $\mathrm{BH}_{2}$, dihydrobiopterin; $\mathrm{BH}_{4}$, tetrahydrobiopterin; EGG, whole eggs; eNOS, endothelial nitric oxide synthase; ET-1, endothelin-1; FL, fluorescence; FMD, flow-mediated dilation; GLU, 100 g glucose; hARG, homoarginine; OGTT, oral glucose tolerance test; PPH, postprandial hyperglycaemia; SDMA, symmetric dimethylarginine; VEF, vascular endothelial function; WHITE, egg whites; YOLK, egg yolks.

* Corresponding author: Professor R. S. Bruno, fax +1 614292 4339, email bruno.27@osu.edu 
target PPH itself and/or limit oxidative stress responses that decrease $\mathrm{NO}^{\bullet}$ bioavailability would be expected to lower CVD risk by protecting against impairments in VEF.

Endothelial cells synthesise $\mathrm{NO}^{\bullet}$ in an endothelial $\mathrm{NO}^{\bullet}$ synthase (eNOS)-dependent manner from L-arginine (ARG) ${ }^{(5)}$. In addition, arginine:glycine amidinotransferase synthesises homoarginine (hARG), which is a structural homologue of ARG that functions as an alternate substrate for $\operatorname{eNOS}^{(9)}$. NO $\mathrm{NO}^{\bullet}$ biosynthesis is therefore dependent, in part, on substrate availability (i.e. ARG and hARG) for binding to eNOS. However, oxidative stress dysregulates ARG metabolism ${ }^{(5)}$. Indeed, it increases arginase-mediated catabolism of $\mathrm{ARG}^{(10)}$ while also upregulating protein methyltransferase activity to methylate ARG to asymmetric and symmetric dimethylarginine (ADMA and SDMA ${ }^{(11)}$. Both ADMA and SDMA compete with ARG for endothelial cell uptake ${ }^{(12,13)}$, whereas ADMA also competitively inhibits eNOS activity ${ }^{(11)}$. In controlled trials, PPH-induced oxidative stress impairs VEF in association with increases in ADMA normalised to ARG (ADMA/ARG), an index that suggests reduced $\mathrm{NO}^{\bullet}$ biosynthesis ${ }^{(4)}$. Oxidative stress also decreases arginine:glycine amidinotransferase activity $^{(14)}$, which is expected to lower hARG levels, an effect that is associated with decreased $\mathrm{VEF}^{(15)}$ and increased CVD risk ${ }^{(9,16)}$

In addition to dysregulating ARG metabolism, other mechanisms exist by which PPH-mediated oxidative stress reduces $\mathrm{NO}^{\bullet}$ biosynthesis. Oxidation of the eNOS cofactor tetrahydrobiopterin $\left(\mathrm{BH}_{4}\right)$ to dihydrobiopterin $\left(\mathrm{BH}_{2}\right)$ results in generation of the superoxide due to eNOS uncoupling ${ }^{(17)}$. In agreement, acute incubation of murine endothelial cells with glucose increases superoxide generation in association with decreased $\mathrm{BH}_{4}$ and increased $\mathrm{BH}_{2}{ }^{(18)}$. Importantly, decreased $\mathrm{BH}_{4}$ redox status (i.e. decreased $\mathrm{BH}_{4} / \mathrm{BH}_{2}$ ) is associated with decreased FMD responses ${ }^{(19)}$, suggesting that $\mathrm{BH}_{4} / \mathrm{BH}_{2}$ helps to maintain endothelial function. Hyperglycaemia-mediated increases in angiotensin-II (Ang-II) $)^{(20)}$ and endothelin-1 (ET-1) ${ }^{(21)}$ may also diminish VEF. In addition to their vasoconstricting activities, Ang-II and ET-1 induce oxidative stress that limits $\mathrm{NO}^{\bullet}$ biosynthesis and endothelial-dependent vasodilation by decreasing eNOS expression and activity ${ }^{(22,23)}$. $\mathrm{PPH}-$ mediated oxidative stress also induces inflammation ${ }^{(24)}$ that exacerbates oxidative stress-mediated depletion of $\mathrm{NO}^{\cdot(25)}$. Thus, PPH is implicated in impairing VEF through several oxidative stress-mediated mechanisms.

Epidemiological evidence supports that PPH is a better predictor of CVD-related mortality compared with fasting glucose ${ }^{(26)}$. Thus, dietary approaches that mitigate PPH-mediated oxidative stress would be expected to lower CVD risk by improving VEF. In support, we have reported in prediabetic men that replacing a portion of an OGTT with isoenergetic amounts of non-carbohydrate containing whole eggs (EGG) or egg whites (WHITE), but not egg yolks (YOLK), alleviates impairments in VEF by limiting $\mathrm{PPH}^{(27)}$. The lack of benefit by the egg yolk-based meal was surprising because, identical to the EGG- and WHITE-based meals, it was $25 \%$ lower in glucose content compared with the OGTT $(100 \mathrm{~g})$. This indicates that simply reducing the glucose content alone is not entirely responsible for the observed vasoprotection mediated by EGG or WHITE ${ }^{(27)}$. Studies in vitro demonstrate that WHITE-derived hydrolysates exhibit free-radical scavenging activity ${ }^{(28)}$ and whole-egg hydrolysates also lower oxidative stress in rodent models ${ }^{(29)}$. Hydrolysates derived from either EGG or WHITE also induce $\mathrm{NO}^{\circ}$-dependent vasorelaxation ${ }^{(29,30)}$. Thus, in the present study, we hypothesised that vasoprotection following co-ingestion of EGG or WHITE with glucose occurred by limiting $\mathrm{PPH}$-mediated oxidative stress to improve $\mathrm{NO}^{\bullet}$ bioavailability. We used archived plasma samples collected as part of a previously reported clinical trial ${ }^{(27)}$ (clinicaltrials.gov; NCT02364570) to test this secondary hypothesis.

\section{Methods}

\section{Chemicals and reagents}

HPLC-grade solvents and the following chemicals were purchased from Fisher Scientific: acetonitrile, ascorbic acid, butanol, citric acid, diethylenetriaminepentaacetic acid, dithioerythritol, dithiothreitol, ethanol, hexane, hydrochloric acid, methanol, methyl tert-butyl ether, o-phthalaldehyde, perchloric acid, sodium hydroxide, tetrahydrofuran and thiobarbituric acid. The following were from Sigma-Aldrich: $\beta$-apo-8-carotenal, biopterin, iodine, monosodium phosphate, potassium iodide, octyl sulphate sodium salt and trichloroacetic acid.

\section{Participants and study design}

The study was conducted according to the guidelines laid down in the Declaration of Helsinki. All procedures were approved by the Institutional Review Board at The Ohio State University (2014H0307), and participants provided written informed consent before enrolling. Complete details of this clinical trial and participant characteristics have been reported previously $^{(27)}$. In brief, participants were prediabetic based on fasting blood glucose $(5 \cdot 6-6.9 \mathrm{mmol} / \mathrm{l})^{(31)}$, normocholesterolemic $(<6.2 \mathrm{mmol} / \mathrm{l})$, weight stable ( $\pm 2 \mathrm{~kg}$ for last 3 months) with a BMI between 25 and $35 \mathrm{~kg} / \mathrm{m}^{2}$, and blood pressure $\leq 140 / 90 \mathrm{mmHg}$. They were non-users of dietary supplements or vasoactive medications ( $>1$-month), non-vegetarians, nonsmokers, engaged in $<7 \mathrm{~h} /$ week of aerobic exercise, consumed $<3$ alcoholic drinks/d and self-reported to be free of CVD. Participants consumed identical egg-free euenergetic diets for $3 \mathrm{~d}$ before each intervention, which was reported previously to be verified from written food records ${ }^{(27)}$.

In this randomised, cross-over clinical trial ${ }^{(27)}$, men $(25-50$ years) with prediabetes completed four postprandial trials that were each separated by $7 \mathrm{~d}$. For the present investigation, changes in plasma total $\mathrm{NO} \bullet$ metabolites (i.e. the sum of nitrate and nitrite), malondialdehyde, antioxidants (vitamins $\mathrm{C}, \mathrm{E}$ and carotenoids), ARG and endogenous metabolites (hARG, ADMA and SDMA), $\mathrm{BH}_{4} / \mathrm{BH}_{2}$, vasoconstrictors (Ang-II and ET-1), inflammatory markers (C-reactive protein (CRP), myeloperoxidase (MPO) and TNF- $\alpha$ ) were evaluated during a 3 -h postprandial period following the ingestion of isoenergetic test meals. Test meals consisted of $100 \mathrm{~g}$ glucose (GLU) alone or $75 \mathrm{~g}$ glucose in combination with $1.5 \mathrm{EGG}$, seven WHITE or two YOLK. Test meals were formulated, as described previously ${ }^{(27)}$, to be similar to the typical energy content of breakfast 
consumed by American males (approximately $1670 \mathrm{~kJ}$ $(400 \mathrm{kcal}))^{(32)}$. In addition, the glucose content in egg-based meals $(75 \mathrm{~g})$ was selected on the basis that it reflects the dose used in an OGTT, which has been shown to also induce PPH, oxidative stress and impair $\mathrm{VEF}^{(4)}$. Plasma collected before $(t=0 \mathrm{~min})$ and at 30, 60, 90, 120, 150 and $180 \mathrm{~min}$ after meal ingestion was used for the analyses in the present study. All assessments were performed from 0 to $180 \mathrm{~min}$ because PPH transiently impairs VEF during this time period in response to an OGTT $^{(4,27)}$. For measurements of biopterin, plasma was obtained by centrifugation from blood collected in EDTA tubes containing $0 \cdot 1 \%(\mathrm{w} / \mathrm{v})$ dithioerythritol and $0 \cdot 1 \%(\mathrm{w} / \mathrm{v})$ ascorbic acid. For measurements of vitamin $\mathrm{C}$ and uric acid, Na heparinised plasma was mixed (1:1) with $10 \%(\mathrm{w} / \mathrm{v})$ perchloric acid containing $1 \mathrm{~mm}$ diethylenetriaminepentaacetic acid and centrifuged $\left(4^{\circ} \mathrm{C}, 5 \mathrm{~min}, 15000 \mathbf{g}\right)$. The supernatant was snapfrozen in liquid $\mathrm{N}_{2}$. All samples were stored at $-80^{\circ} \mathrm{C}$ until analysis.

\section{Oxidative stress and antioxidant measurements}

Plasma malondialdehyde was measured by HPLC-fluorescence (FL) following thiobarbituric acid derivatisation and butanol extraction as we described $^{(4)}$ on a Shimadzu LC-20XR system equipped with a RF-20AXL FL detector set to $532 / 553 \mathrm{~nm}$ (excitation/emission). Plasma ascorbic acid and uric acid were measured from perchloric acid-treated plasma, and vitamin $\mathrm{E}$ (as $\alpha$ - and $\gamma$-tocopherol) was measured following saponification and hexane extraction using our separate HPLCelectrochemical detection procedures ${ }^{(33)}$. Plasma carotenoids were measured as described ${ }^{(34)}$ with minor modification. In brief, $200 \mu$ of plasma was mixed with $200 \mu$ l of ethanol, $50 \mu \mathrm{l}$ of $\beta$-apo-8-carotenal ( $60 \mathrm{~nm}$, internal standard) before being extracted three times with $3 \mathrm{ml}$ of methanol-tetrahydrofuran (1:1) and $4 \mathrm{ml}$ of hexane. The extract was evaporated under $\mathrm{N}_{2}$ gas and reconstituted in $150 \mu \mathrm{l}$ methanol and $150 \mu \mathrm{l}$ methyl tert-butyl ether before being filtered $(0.22 \mu \mathrm{m})$ and injected $(50 \mu \mathrm{l})$ onto the HPLC system. Carotenoids (lutein, zeaxanthin, $\alpha$ - and $\beta$-carotene, $\alpha$ - and $\beta$-cryptoxanthin, lycopene) were quantified at $450 \mathrm{~nm}$ on a Waters 2695 HPLC system equipped with a 2996 photodiode array detector. Separation was performed isocratically $(1.0 \mathrm{ml} / \mathrm{min})$ using a YMC C30 column $(150 \times 4.6 \mathrm{~mm}, 3.0 \mu \mathrm{m})$ and a mobile phase consisting of methanol-methyl tert-butyl ether-water (96:2:2).

\section{Measurements of nitric oxide homeostasis}

Plasma total nitrite and nitrate, the stable end-products of $\mathrm{NO}^{\bullet(10)}$, were assessed by spectrophotometry to estimate $\mathrm{NO}^{\bullet}$ status in accordance with the manufacturer's instructions (Cayman Chemical). Plasma ARG and its metabolites (ADMA, SDMA and hARG) were measured by HPLC-FL $(340 / 455 \mathrm{~nm}$, excitation/emission) following $O$-phthalaldehyde derivatisation on the aforementioned Shimadzu HPLC-FL system as we described $^{(4)}$.

Total biopterin represents the sum of $\mathrm{BH}_{4}, \mathrm{BH}_{2}$ and biopterin and is determined using iodine oxidation under acidic and alkaline conditions ${ }^{(35)}$. Under acidic conditions, both $\mathrm{BH}_{4}$ and
$\mathrm{BH}_{2}$ are oxidised to biopterin, whereas only $\mathrm{BH}_{2}$ is oxidised to biopterin under basic conditions. Thus, the difference in biopterin concentrations between acidic and basic oxidation represents $\mathrm{BH}_{4}$ levels ${ }^{(35)}$. Plasma biopterin was measured as described $^{(35)}$ with minor modification. In brief, plasma $(400 \mu \mathrm{l})$ was mixed with $1 \mathrm{~m}$ trichloroacetic acid and centrifuged $\left(4^{\circ} \mathrm{C}\right.$, $15 \mathrm{~min}, 20000 \mathrm{~g}$ ). Following centrifugation, two aliquots of supernatant $(100 \mu \mathrm{l}$ each) were used to evaluate biopterin levels following acidic and basic oxidation. For acidic oxidation, $100 \mu \mathrm{l}$ $0.2 \mathrm{~m}$ hydrochloric acid and $10 \mu \mathrm{l}$ iodine solution ( $0.9 \%(\mathrm{w} / \mathrm{v})$ iodine and $1.8 \%(\mathrm{w} / \mathrm{v})$ potassium iodide in $0.1 \mathrm{~m}$ hydrochloric acid) were mixed with supernatant. For basic oxidation, $100 \mu \mathrm{l}$ $0 \cdot 2 \mathrm{~m}$ sodium hydroxide and $10 \mu \mathrm{l}$ iodine solution $(0.9 \%(\mathrm{w} / \mathrm{v})$ iodine and $1.8 \%(\mathrm{w} / \mathrm{v})$ potassium iodide in $0.1 \mathrm{~m}$ sodium hydroxide) were mixed with supernatant. All samples were then incubated in the dark at room temperature for $60 \mathrm{~min}$ and subsequently mixed with $10 \mu \mathrm{l} 2 \%(\mathrm{w} / \mathrm{v})$ ascorbic acid to reduce excess iodine. Samples were maintained in a refrigerated autosampler $\left(10^{\circ} \mathrm{C}\right)$ and injected $(30 \mu \mathrm{l})$ onto the aforementioned Waters HPLC system equipped with a $474 \mathrm{FL}$ detector $\left(350 / 450 \mathrm{~nm}\right.$, excitation/emission) as described ${ }^{(36)}$, with minor modification. Separation was performed isocratically $(0.9 \mathrm{ml} / \mathrm{min})$ on a Phenomenex Kinetex Evo C18 column $(250 \times 4.6 \mathrm{~mm}, 5.0 \mu \mathrm{m})$ thermostated to $35^{\circ} \mathrm{C}$ using a mobile phase composed of $6.5 \mathrm{~mm}$ monosodium phosphate, $6 \mathrm{~mm}$ citric acid, $1 \mathrm{~mm}$ octyl sulphate sodium salt, $2.5 \mathrm{~mm}$ diethylenetriaminepentaacetic acid, $1 \mathrm{~mm}$ dithiothreitol and $2 \%$ acetonitrile ( $\mathrm{pH} 3 \cdot 0)$. Quantification was performed using external standards that were prepared in parallel.

\section{Vasoconstrictors and inflammatory markers}

Plasma vasoconstrictors (Ang-II and ET-1) were measured by ELISA (Enzo Life Sciences Inc.) according to the manufacturers' instructions using a Synergy $\mathrm{H} 1$ microplate reader (Biotek Instruments). Plasma CRP and MPO (BioCheck Inc.) and TNF- $\alpha$ (R\&D Systems Inc.) were also measured by ELISA.

\section{Statistical methods}

The parent study ${ }^{(27)}$ was powered to detect between-treatment differences in postprandial FMD responses (primary outcome variable; clinicaltrials.gov: NCT02364570), with a power calculation indicating that nine subjects would be needed to reject the null hypothesis with $90 \%$ power $(P<0.05)$. The present study, which utilises archived specimens from these same participants, was powered to detect differences in the lipid peroxidation biomarker malondialdehyde. This biomarker was chosen to test the secondary hypothesis that previously observed improvements in VEF (i.e. FMD) in relation to PPH would be partly attributed to an egg-mediated attenuation in oxidative stress. Although no postprandial studies have examined egg-based meals on malondialdehyde responses, we reported that postprandial malondialdehyde is highly correlated with glycaemic responses $(r 0.87 ; P<0.05)^{(4)}$. For the present study, based on EGG attenuating glycaemia by approximately $50 \%$ compared with GLU, we predicted an approximately $50 \%$ reduction in malondialdehyde. Thus, a minimum of seventeen 
subjects would be needed to detect statistically significant between-treatment effects with $80 \%$ power $(P<0.05)$, thereby indicating that our enrollment of twenty participants ${ }^{(27)}$ was sufficient to appropriately test our secondary hypothesis. Data reported (means with their standard errors) are change $(\Delta)$ from baseline $(t=0 \mathrm{~min})$ to visualise between-treatment effects for postprandial responses and were analysed using GraphPad Prism (version 7). Time, treatment and time $\times$ treatment interaction effects were evaluated using two-way repeated-measures (RM) ANOVA with the Bonferroni's correction. Postprandial AUC $\left(\mathrm{AUC}_{0-180 \mathrm{~min}}\right)$, as the net difference between responses going above (i.e. positive) and below (i.e. negative) baseline, was calculated using the trapezoidal rule. Differences among trials for baseline values as well as $\mathrm{AUC}_{0-180}$ min were assessed using one-way RM ANOVA with the Bonferroni's post-test. Correlation coefficients $(r$ ) between AUC among study variables were calculated using multiple linear regression while controlling for within-subject $\mathrm{RM}^{(37)} . P<0.05$ was considered statistically significant.

\section{Results}

\section{Participants and postprandial glycaemic and vascular} responses

Complete details of participant characteristics and postprandial glycaemic and FMD responses were reported previously ${ }^{(27)}$. In brief, men were prediabetic with a fasting glucose of 6.0 (SEM $0 \cdot 1) \mathrm{mmol} / \mathrm{l}$, non-hypertensive (123 (SEM 4)/72 (SEM 6) $\mathrm{mmHg}$ ) and normocholesterolemic (5.0 (SEM $0 \cdot 1) \mathrm{mmol} / \mathrm{l})$. They were obese on the basis of mean BMI $\left(31.1(\operatorname{sem~} 0.7) \mathrm{kg} / \mathrm{m}^{2}\right)$, but five of the twenty participants had a BMI indicative of overweightness.

Compared with the GLU trial, postprandial increases in glucose and decreases in FMD were attenuated in the EGG and WHITE trials, but not in the YOLK trial ${ }^{(27)}$. Relative to baseline (0 min), plasma glucose concentrations increased at 30-120 min in GLU and YOLK whereas they only increased at 30-90 min in EGG and WHITE. FMD responses decreased at 30-60 min in GLU and at 30-180 min in YOLK, but only at $30 \mathrm{~min}$ in EGG and WHITE. Compared with GLU, the AUC of glucose and FMD were similarly attenuated in the EGG and WHITE trials; the YOLK trial was not different from the GLU trial. Plasma archived from the previously conducted clinical trial ${ }^{(27)}$ was used to test the current hypothesis that EGG and WHITE protected against PPH-mediated impairments in VEF by attenuating oxidative stress responses that otherwise decrease $\mathrm{NO}^{\bullet}$ bioavailability. In brief, none of the plasma biomarkers reported below differed at baseline $(t=0)$ among trials $(P>0.05$; Table 1$)$ with the exception of Ang-II, which was lower in EGG compared with GLU.

\section{Plasma malondialdehyde}

The magnitude of PPH is correlated with increases in oxidative stress $^{(4)}$. Following test meal ingestion, we observed time, treatment and time $\times$ treatment effects for malondialdehyde

Table 1. Baseline values for plasma biomarkers from each intervention arm*

(Mean values with their standard errors, $n$ 20)

\begin{tabular}{|c|c|c|c|c|c|c|c|c|}
\hline & \multicolumn{2}{|c|}{ GLU } & \multicolumn{2}{|c|}{$E G G$} & \multicolumn{2}{|c|}{ WHITE } & \multicolumn{2}{|c|}{ YOLK } \\
\hline & Mean & SEM & Mean & SEM & Mean & SEM & Mean & SEM \\
\hline MDA $(\mu \mathrm{mol} / \mathrm{l})$ & $1 \cdot 2$ & 0.05 & 1.3 & 0.09 & $1 \cdot 1$ & 0.04 & $1 \cdot 1$ & 0.04 \\
\hline $\mathrm{NOx}(\mu \mathrm{mol} / \mathrm{l})$ & $36 \cdot 4$ & $4 \cdot 4$ & $34 \cdot 4$ & 3.5 & $31 \cdot 3$ & $2 \cdot 8$ & $32 \cdot 6$ & $3 \cdot 3$ \\
\hline $\mathrm{ARG}(\mu \mathrm{mol} / \mathrm{l})$ & $96 \cdot 4$ & $4 \cdot 7$ & $95 \cdot 3$ & 3.7 & 99.9 & $5 \cdot 4$ & $98 \cdot 0$ & $5 \cdot 3$ \\
\hline hARG $(\mu \mathrm{mol} / \mathrm{l})$ & 3.7 & 0.2 & $3 \cdot 8$ & 0.2 & $3 \cdot 7$ & 0.2 & 3.6 & 0.2 \\
\hline ADMA (nmol/l) & $536 \cdot 6$ & $24 \cdot 2$ & 561.0 & $30 \cdot 5$ & $557 \cdot 8$ & $29 \cdot 0$ & 543.8 & $32 \cdot 1$ \\
\hline SDMA (nmol/l) & $459 \cdot 2$ & $18 \cdot 8$ & $466 \cdot 6$ & $22 \cdot 2$ & $468 \cdot 8$ & 20.9 & 453.7 & 21.4 \\
\hline $\mathrm{BH}_{4}(\mathrm{nmol} / \mathrm{l})$ & 4.6 & 0.7 & $5 \cdot 8$ & $1 \cdot 1$ & 3.9 & 0.5 & $5 \cdot 1$ & 0.8 \\
\hline $\mathrm{BH}_{2}(\mathrm{nmol} / \mathrm{l})$ & 1.9 & 0.3 & $2 \cdot 3$ & 0.3 & $1 \cdot 8$ & 0.2 & 1.8 & $0 \cdot 2$ \\
\hline Ang-II (pg/ml) & $13 \cdot 0^{a}$ & 0.5 & $11 \cdot 7^{b}$ & 0.4 & $12 \cdot 1^{a, b}$ & 0.4 & $12 \cdot 3^{a, b}$ & 0.6 \\
\hline ET-1 (pg/ml) & $1 \cdot 31$ & 0.10 & 1.56 & 0.13 & 1.56 & 0.14 & 1.47 & 0.11 \\
\hline CRP (mg/l) & 1.57 & 0.29 & 1.58 & 0.34 & $1 \cdot 39$ & 0.22 & 1.55 & $0 \cdot 26$ \\
\hline TNF- $a(\mathrm{pg} / \mathrm{ml})$ & 0.66 & 0.08 & 0.81 & 0.10 & 0.80 & 0.11 & 0.74 & 0.12 \\
\hline MPO (ng/ml) & 4.96 & 0.28 & $5 \cdot 23$ & 0.37 & $4 \cdot 81$ & 0.38 & $4 \cdot 86$ & 0.32 \\
\hline Vitamin $C(\mu \mathrm{mol} / \mathrm{l})$ & $40 \cdot 7$ & $5 \cdot 2$ & $39 \cdot 0$ & $5 \cdot 0$ & $37 \cdot 7$ & 2.9 & $41 \cdot 2$ & $5 \cdot 5$ \\
\hline Uric acid $(\mu \mathrm{mol} / \mathrm{l})$ & $353 \cdot 1$ & $9 \cdot 9$ & $352 \cdot 8$ & $7 \cdot 7$ & 344.0 & $8 \cdot 9$ & $367 \cdot 7$ & $5 \cdot 5$ \\
\hline$a$-Tocopherol/total lipid ( $\mu \mathrm{mol} / \mathrm{mmol})$ & 3.60 & $0 \cdot 16$ & 3.59 & 0.15 & 3.56 & $0 \cdot 12$ & 3.53 & $0 \cdot 15$ \\
\hline$\gamma$-Tocopherol/total lipid $(\mu \mathrm{mol} / \mathrm{mmol})$ & 0.35 & 0.04 & 0.38 & 0.05 & 0.39 & 0.05 & 0.35 & 0.04 \\
\hline a-Carotene/total lipid ( $\mathrm{nmol} / \mathrm{mmol})$ & $8 \cdot 12$ & 0.91 & 5.99 & 0.65 & $6 \cdot 61$ & 0.83 & $5 \cdot 62$ & 0.47 \\
\hline$\beta$-Carotene/total lipid ( $\mathrm{nmol} / \mathrm{mmol})$ & $28 \cdot 6$ & $2 \cdot 6$ & $24 \cdot 6$ & $3 \cdot 0$ & $26 \cdot 7$ & 2.9 & $27 \cdot 4$ & $3 \cdot 4$ \\
\hline a-Cryptoxanthin/total lipid (nmol/mmol) & $13 \cdot 5$ & $1 \cdot 2$ & 11.9 & $1 \cdot 1$ & $11 \cdot 6$ & $1 \cdot 1$ & $11 \cdot 6$ & $1 \cdot 2$ \\
\hline$\beta$-Cryptoxanthin/total lipid (nmol/mmol) & $11 \cdot 2$ & 0.8 & $9 \cdot 9$ & $1 \cdot 0$ & $8 \cdot 5$ & 0.6 & $9 \cdot 0$ & 0.9 \\
\hline Lutein/total lipid (nmol/mmol) & $16 \cdot 3$ & $2 \cdot 3$ & $15 \cdot 6$ & $2 \cdot 2$ & $14 \cdot 7$ & 1.9 & $17 \cdot 6$ & $2 \cdot 6$ \\
\hline Lycopene/total lipid (nmol/mmol) & $128 \cdot 3$ & $12 \cdot 0$ & $119 \cdot 0$ & $13 \cdot 4$ & $117 \cdot 6$ & $13 \cdot 7$ & $115 \cdot 9$ & $13 \cdot 5$ \\
\hline Zeaxanthin/total lipid (nmol/mmol) & 4.93 & 0.66 & $4 \cdot 86$ & 0.70 & $4 \cdot 64$ & 0.72 & $5 \cdot 53$ & 0.87 \\
\hline
\end{tabular}

GLU, $100 \mathrm{~g}$ glucose; EGG, whole eggs; WHITE, egg whites; YOLK, egg yolks; MDA, malondialdehyde; NOx, nitric oxide metabolites (nitrate/nitrite); ARG, arginine; hARG, homoarginine; ADMA, asymmetric dimethylarginine; SDMA, symmetric dimethylarginine; $\mathrm{BH}_{4}$, tetrahydrobiopterin; $\mathrm{BH}_{2}$, dihydrobiopterin; Ang-II, angiotensin-II; $\mathrm{ET}-1$, endothelin-1; CRP, C-reactive protein; MPO, myeloperoxidase.

a,b Mean values with unlike superscript letters were significantly different. $P>0.05$ for all data reported except for Ang-II $(P=0.03)$.

* Total lipid equals the sum of total cholesterol and TAG. 
( $P<0.001$; Fig. 1). Relative to 0 min, malondialdehyde was increased at 30-120 min in the GLU and YOLK trials but only at 30-60 min in the EGG trial and at 30-90 min in the WHITE trial. Compared with GLU, malondialdehyde was increased to a lesser extent at 60-180 $\mathrm{min}$ in EGG and at $60-120 \mathrm{~min}$ in WHITE, whereas it was not different in YOLK. AUC was also lower $(P<0 \cdot 001)$ in the EGG and WHITE trials compared with the GLU and YOLK trials; the YOLK trial was not different from the GLU trial. The AUC of malondialdehyde was positively correlated $(P<0.05$; Table 2$)$ with glucose and negatively correlated with FMD $(P=0 \cdot 01)$. Data suggest that the co-ingestion
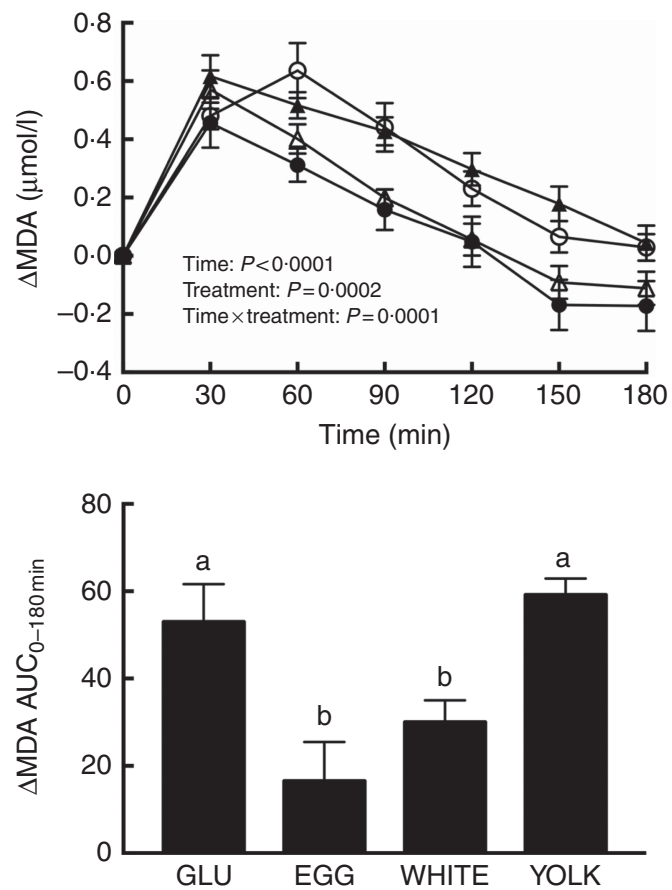

Fig. 1. Postprandial malondialdehyde (MDA) responses and $A \cup C_{0-180}$ min following ingestion of glucose in the absence or presence of egg-based meals by prediabetic men. Postprandial responses were analysed using two-way repeated-measures (RM) ANOVA with Bonferroni's post hoc test. $\mathrm{AUC}_{0-180}$ min was calculated using the trapezoidal rule and analysed using one-way RM ANOVA with Bonferroni's post hoc test. Values are means $(n 20)$, with their standard errors represented by vertical bars. ${ }^{a, b}$ Mean values with unlike letters were significantly different $(P<0.05)$. GLU $(-\bigcirc-100 \mathrm{~g}$ glucose; EGG (- - ), whole eggs; WHITE $(\triangle)$ ), egg whites; YOLK $(\multimap)$, egg yolks. of EGG or WHITE with glucose protects against PPH-mediated decreases in vascular function by limiting lipid peroxidation.

\section{Plasma antioxidants}

Plasma antioxidants were assessed to evaluate the extent to which test meals regulated postprandial oxidative stress by altering antioxidant status. Only time effects were observed for postprandial changes in vitamin $\mathrm{C}$ and uric acid $(P<0.0001$, data not shown). Specifically, vitamin $C$ decreased at 30$180 \mathrm{~min}$ and uric acid at $150-180 \mathrm{~min}$ regardless of trial. However, the AUC of vitamin $\mathrm{C}$ and uric acid did not differ among treatments $(P>0 \cdot 05$, online Supplementary Table S1). We also assessed lipophilic antioxidants, specifically vitamin $\mathrm{E}$ and a panel of carotenoids. Because participants' lipid responses decreased postprandially regardless of treatment ${ }^{(27)}$, lipophilic antioxidant concentrations were normalised to total lipid (i.e. the sum of total cholesterol and TAG) as suggested ${ }^{(38)}$. We observed that $\gamma$-tocopherol decreased at 120-180 min relative to 0 min $(P<0.0001$, data not shown) but its AUC as well as that of $\alpha$-tocopherol did not differ among trials $(P>0 \cdot 05$, online Supplementary Table S1). Of the seven carotenoids evaluated (lutein, zeaxanthin, $\alpha$ - and $\beta$-carotene, $\alpha$ - and $\beta$-cryptoxanthin, lycopene), we only detected a time effect for $\beta$-cryptoxanthin and a treatment effect for lutein. $\beta$-Cryptoxanthin was increased at $180 \mathrm{~min}$ relative to $0 \mathrm{~min}(P<0 \cdot 01$, data not shown). Lutein was significantly lower in YOLK at $30 \mathrm{~min}$ and at $120-150 \mathrm{~min}$ compared with EGG and at 30-180 min compared with WHITE ( $P=0 \cdot 01$, data not shown). However, the AUC of lutein showed no treatment differences despite a significant main effect ( $P=0.02$, online Supplementary Table S1). Thus, it is likely that EGG- and WHITE-based test meals attenuated lipid peroxidation independent of improved antioxidant status.

\section{Plasma nitric oxide metabolites}

FMD largely reflects $\mathrm{NO}^{\bullet}$-dependent vasodilation ${ }^{(7)}$, suggesting that improvements in vascular function by EGG and WHITE were attributed to greater $\mathrm{NO}^{\bullet}$ bioavailability. Plasma $\mathrm{NO}^{\bullet}$ metabolites (i.e. the sum of nitrite and nitrate), an index of $\mathrm{NO}^{\bullet}$ bioavailability, showed main and interactive effects $(P \leq 0 \cdot 01$; Fig. 2$)$. Relative to 0 min, NO metabolites were lower at $60-180 \mathrm{~min}$ in the GLU trial but were not different from $0 \mathrm{~min}$ in response to any egg-based meals. Compared with GLU, NO metabolites decreased to a lesser extent at 60-150 min in the EGG trial, at 60-90 and $150 \mathrm{~min}$ in the

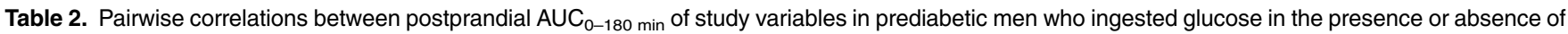
egg-based meals* $† \ddagger$

\begin{tabular}{lcccccccccc}
\hline & Glucose & MDA & NOx & ARG & hARG & ADMA/ARG & SDMA/ARG & BH $_{4} / \mathrm{BH}_{2}$ & Ang-II & ET-1 \\
\hline FMD & NS & $-0.39 \ddagger$ & $0.41 \ddagger$ & $0.38 \ddagger$ & $0.30 \dagger$ & $-0.34 \ddagger$ & $-0.40 \ddagger$ & NS & NS & NS \\
Glucose & & $0.36 \ddagger$ & NS & NS & NS & $0.34 \ddagger$ & $0.29 \dagger$ & NS & NS & $0.29 \dagger$ \\
MDA & & & $-0.31 \dagger$ & NS & NS & $0.29 \dagger$ & NS & NS & NS & NS \\
NOx & & & & $0.42 \ddagger$ & $0.29 \dagger$ & $-0.40 \ddagger$ & $-0.45 \ddagger$ & NS & $-0.38 \ddagger$ & NS \\
ARG & & & & & $0.57 \ddagger$ & $-0.77 \ddagger$ & $-0.74 \ddagger$ & NS & $-0.45 \ddagger$ & NS \\
\hline
\end{tabular}

MDA, malondialdehyde; NOx, nitric oxide metabolites (nitrate/nitrite); ARG, arginine; hARG, homoarginine; ADMA, asymmetric dimethylarginine; SDMA, symmetric dimethylarginine; $\mathrm{BH}_{4}$, tetrahydrobiopterin; $\mathrm{BH}_{2}$, dihydrobiopterin; Ang-II, angiotensin-II; ET-1, endothelin-1; FMD, flow-mediated dilation.

* Numerical data represent correlation coefficients $(r)$ between study variables.

$+P<0.05$.

$\ddagger P \leq 0.01$. 

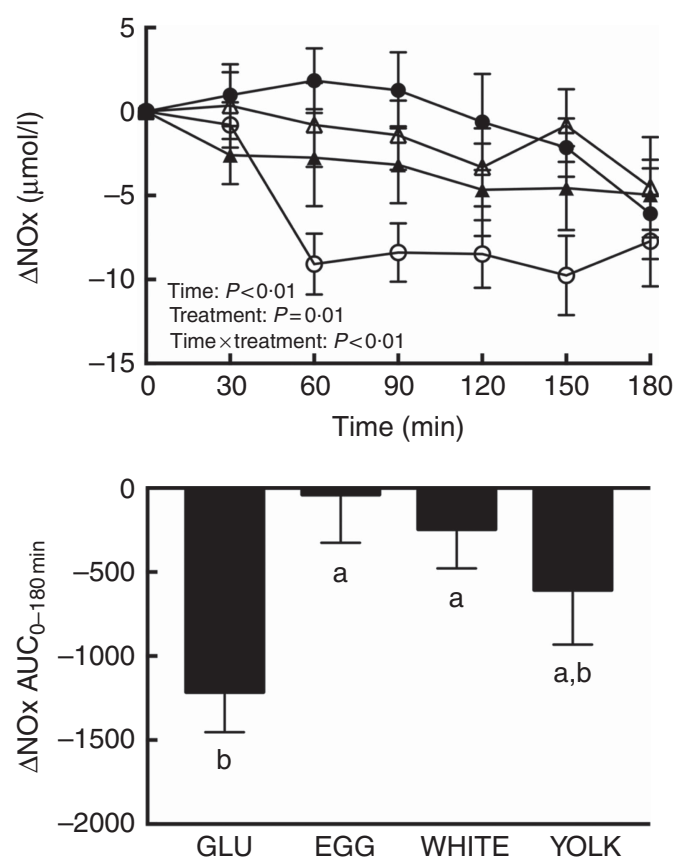

Fig. 2. Postprandial nitric oxide metabolites (nitrate/nitrite) (NOx) responses and $\mathrm{AUC}_{0-180 \mathrm{~min}}$ following ingestion of glucose in the absence or presence of eggbased meals by prediabetic men. Postprandial responses were analysed using two-way repeated-measures (RM) ANOVA with Bonferroni's post hoc test. $\mathrm{AUC}_{0}$ $180 \mathrm{~min}$ was calculated using the trapezoidal rule and analysed using one-way RM ANOVA with Bonferroni's post hoc test. Values are means ( $n$ 20), with their standard errors represented by vertical bars. ${ }^{\mathrm{a}, \mathrm{b}}$ Mean values with unlike letters were significantly different $(P<0.05)$. GLU (- -100 g glucose; EGG (- - ), whole eggs; WHITE ( $\triangle$ - ) , egg whites; YOLK ( $\longrightarrow-$ ), egg yolks.
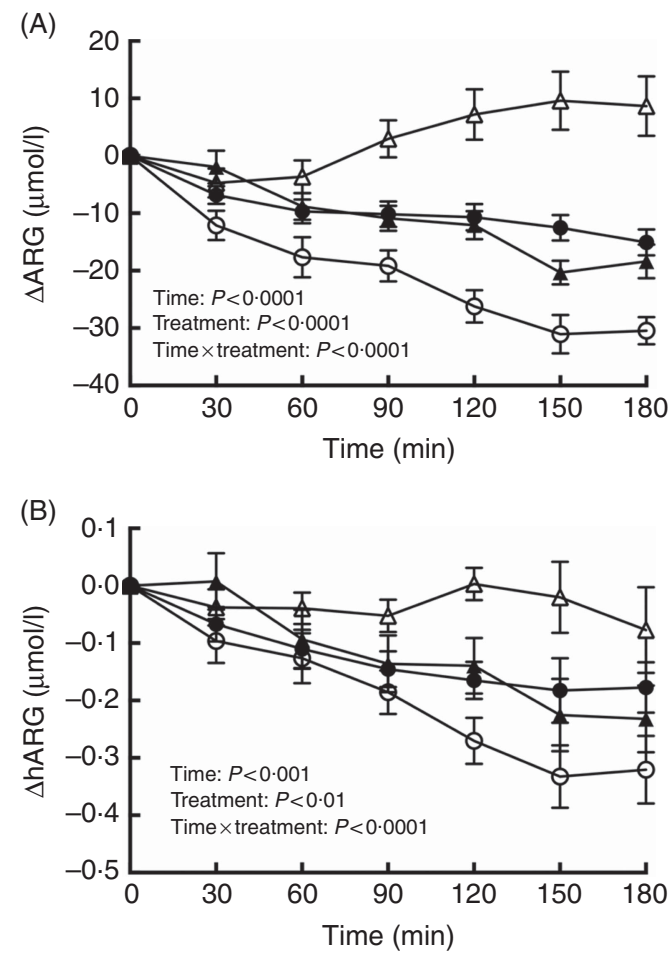

WHITE trial, but only at 60 min in the YOLK trial. AUC was similarly greater in the EGG and WHITE trials compared with the GLU trial $(P<0 \cdot 01)$, whereas the YOLK trial was not different from the GLU trial $(P>0 \cdot 05)$. NO ${ }^{\bullet}$ metabolites positively correlated with FMD $(P<0 \cdot 01$, Table 2$)$, suggesting that improved VEF was due to greater $\mathrm{NO}^{\bullet}$ bioavailability. An inverse relationship between $\mathrm{NO}^{\bullet}$ metabolites and malondialdehyde $(P<0.05)$ was also observed, consistent with oxidative stress diminishing $\mathrm{NO}^{\bullet}$ bioavailability. Data suggest that EGG and WHITE protect against impairments in $\mathrm{VEF}$, at least in part, by preserving $\mathrm{NO}^{\circ}$ bioavailability that is otherwise decreased by PPH-mediated oxidative stress.

\section{L-Arginine and homoarginine availability}

Postprandial changes in ARG and hARG were assessed to test the hypothesis that improved $\mathrm{NO}^{\bullet}$ status during EGG and WHITE (Fig. 2) was attributed to greater substrate availability. We observed main and interactive effects for both ARG and hARG $(P<0.01$; Fig. 3(A) and (B)). Relative to 0 min, ARG was decreased at 30-180 $\mathrm{min}$ in the GLU trial, at 60-180 $\mathrm{min}$ in the EGG trial and at 90-180 $\mathrm{min}$ in the YOLK trial (Fig. 3(A)). However, ARG was increased at $150 \mathrm{~min}$ in the WHITE trial. Decreases in ARG otherwise occurring in GLU were attenuated at 60-180 min in EGG and at 30-180 min in YOLK. In addition, ARG was increased at 90-180 min in WHITE compared with GLU. The AUC of ARG was similarly greater in EGG and YOLK, but even greater in WHITE $(P<0 \cdot 0001)$. ARG AUC $_{\text {was }}$ also positively correlated with $\mathrm{NO}^{\bullet}$ metabolites and FMD $(P<0 \cdot 01$; Table 2).
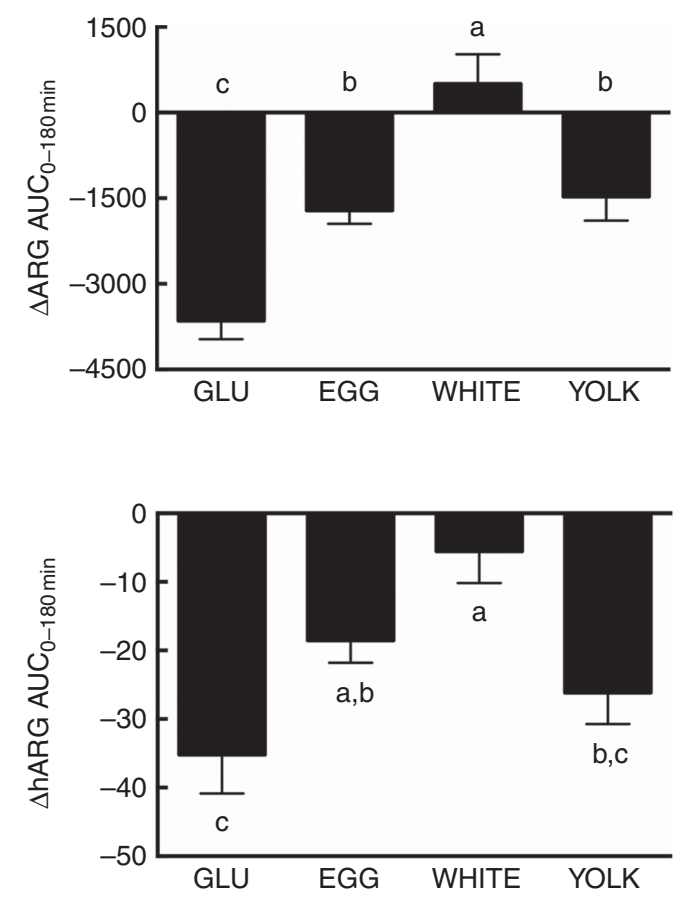

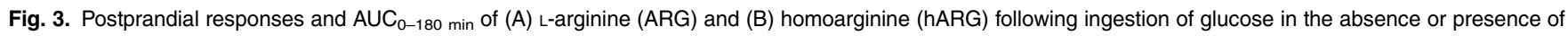
egg-based meals by prediabetic men. Postprandial responses were analysed using two-way repeated-measures (RM) ANOVA with Bonferroni's post hoc test. AUC $\mathrm{A}_{-}$ $180 \mathrm{~min}$ was calculated using the trapezoidal rule and analysed using one-way RM ANOVA with Bonferroni's post hoc test. Values are means ( $n$ 20), with their standard

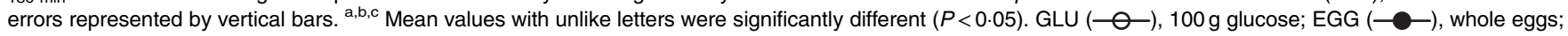
WHITE ( $\triangle$ ), egg whites; YOLK ( $\longrightarrow-)$, egg yolks. 
Relative to baseline levels, hARG was decreased at 90$180 \mathrm{~min}$ in the GLU, EGG and YOLK trials but were unaffected in the WHITE trial (Fig. 3(B)). Compared with GLU, decreases in hARG were attenuated at $120 \mathrm{~min}$ in YOLK, at $150-180 \mathrm{~min}$ in EGG and at $90-180 \mathrm{~min}$ in WHITE. The AUC of hARG was greater in EGG and greatest in WHITE compared with GLU $(P=0.0001)$; YOLK did not differ from GLU $(P>0.05)$. The AUC of hARG was positively correlated $(P<0.05$, Table 2$)$ with the AUC of ARG, NO metabolites and FMD. Thus, co-ingestion of EGG or WHITE with glucose may in part protect against declines in $\mathrm{NO}^{\circ}$ bioavailability by improving substrate availability for eNOS.

\section{Competitive inhibition of endothelial nitric oxide synthase activity and L-arginine uptake}

We assessed changes in the ARG metabolites ADMA and SDMA to consider the possibility that greater $\mathrm{NO}^{\bullet}$ metabolites during the EGG and WHITE trials were attributed to limiting competitive inhibition of eNOS by $\mathrm{ADMA}^{(11)}$ and arginine uptake by ADMA and SDMA ${ }^{(12,13)}$. For ADMA/ARG as well as SDMA/ARG, we observed effects due to time, treatment and their interaction $(P<0.0001$; Fig. 4(A) and (B)). ADMA/ARG (Fig. 4(A)) was increased at 30-180 min in the GLU and YOLK trials and at 30$60 \mathrm{~min}$ in the EGG trial relative to $0 \mathrm{~min}$. ADMA/ARG was also increased at $30 \mathrm{~min}$ in the WHITE trial and decreased relative to baseline at 120-180 min. ADMA/ARG increased to a lesser extent at 60-180 $\mathrm{min}$ in the WHITE trial and at 90-180 $\mathrm{min}$ in the
EGG and YOLK trials compared with GLU. Likewise, the AUC of ADMA/ARG was lower in EGG and YOLK and lowest in WHITE compared with GLU $(P<0 \cdot 0001)$. ADMA/ARG AUC $_{\text {Was }}$ inversely correlated $(P \leq 0 \cdot 01$; Table 2$)$ with AUC of $\mathrm{NO}^{\bullet}$ metabolites, ARG and FMD, but positively correlated $(P<0.05)$ with AUC of glucose and malondialdehyde. This suggests that the observed vasoprotection in the EGG and WHITE trials was attributed to, at least in part, greater $\mathrm{NO}^{\bullet}$ bioavailability that was otherwise reduced by competitive inhibition of eNOS.

Relative to $0 \mathrm{~min}$, the SDMA:ARG ratio (Fig. 4(B)) increased at 30-180 min in the GLU trial, at 120-180 min in the EGG trial and at 60-180 min in the YOLK trial, whereas it decreased at 120$180 \mathrm{~min}$ in the WHITE trial. Relative to the GLU trial, SDMA/ ARG was attenuated at 30-180 min in the WHITE trial, at 60$180 \mathrm{~min}$ in the EGG trial and at 90-180 min in the YOLK trial. The SDMA/ARG ${ }_{\mathrm{AUC}}$ was also similarly lower in the EGG and YOLK trials but lowest in the WHITE trial $(P<0 \cdot 0001)$ compared with the GLU trial. SDMA/ARG AUC $_{\text {was inversely corre- }}$ lated $(P<0 \cdot 01$; Table 2$)$ with AUC of NO ${ }^{\bullet}$ metabolites, ARG and

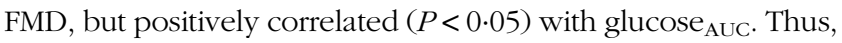
improvements in $\mathrm{NO}^{\circ}$ bioavailability and vascular function by EGG and WHITE may occur, in part, by improving ARG uptake into endothelial cells.

\section{Tetrahydrobiopterin redox status}

Postprandial $\mathrm{BH}_{4}$ redox status was assessed to determine if improved $\mathrm{NO}^{\bullet}$ status in the EGG and WHITE trials was
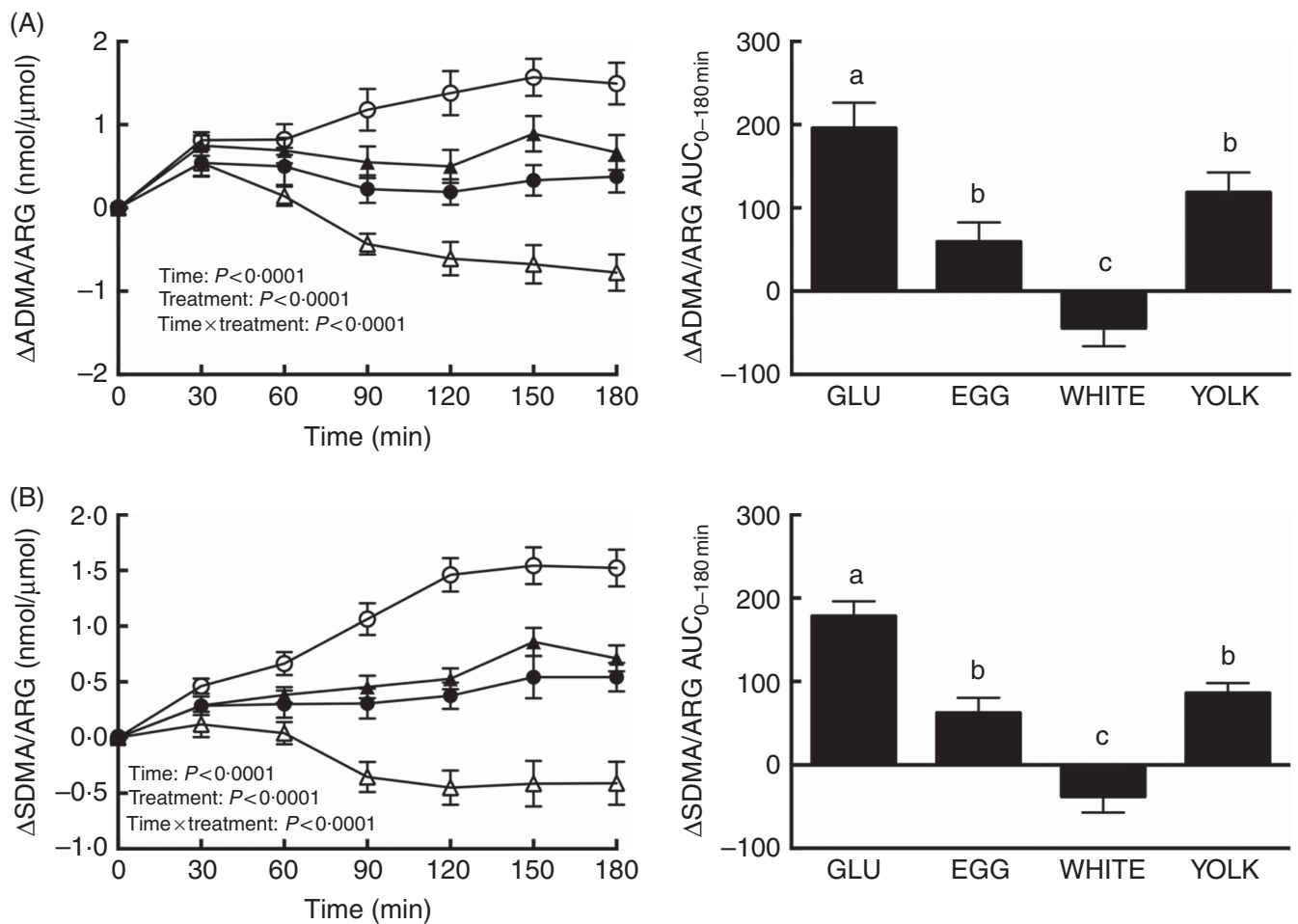

Fig. 4. Postprandial responses and $A U C_{0-180}$ min of $(A)$ asymmetric dimethylarginine (ADMA)/L-arginine (ARG) and (B) symmetric dimethylarginine (SDMA)/ARG following ingestion of glucose in the absence or presence of egg-based meals by prediabetic men. Postprandial responses were analysed using two-way repeatedmeasures (RM) ANOVA with Bonferroni's post hoc test. AUC $\mathrm{C}_{0-180}$ min was calculated using the trapezoidal rule and analysed using one-way RM ANOVA with Bonferroni's post hoc test. Values are means $(n 20)$, with their standard errors represented by vertical bars. ${ }^{a, b, c}$ Mean values with unlike letters were significantly

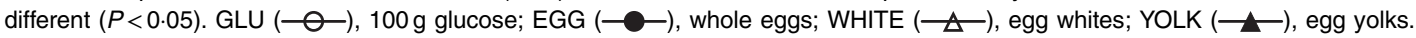


attributed to attenuating eNOS uncoupling ${ }^{(18)}$. However, changes in plasma concentrations of $\mathrm{BH}_{4}, \mathrm{BH}_{2}$ and $\mathrm{BH}_{4} / \mathrm{BH}_{2}$ and their respective AUC did not differ among trials $(P>0.05$, online Supplementary Fig. S1).

\section{Plasma vasoconstrictors and inflammatory markers}

Ang-II and ET-1 and inflammatory markers were assessed postprandially to define their potential contribution to reduce $\mathrm{NO}^{\circ}$ bioavailability. We observed time, treatment and interaction effects for Ang-II ( $P<0.0001$; Fig. 5(A)). Relative to $0 \mathrm{~min}$, Ang-II increased at 30-180 min in the GLU trial but only at 60$120 \mathrm{~min}$ in all egg-based trials. GLU-induced increases in Ang-II were similarly attenuated at 30-180 min in egg-based trials. The AUC of Ang-II was also similarly lower in egg-based treatments compared with the GLU trial $(P<0 \cdot 0001)$. Ang-II ${ }_{\mathrm{AUC}}$ negatively correlated $(P<0 \cdot 01$; Table 2$)$ with AUC of NO ${ }^{\bullet}$ metabolites and ARG.

Plasma ET-1 showed an effect due to treatment only $(P=0.03$; Fig. 5(B)). Although plasma ET-1 increased to the greatest extent in the GLU trial, its concentrations were attenuated at 30-60 min in the WHITE trial and at 30-90 and $180 \mathrm{~min}$ in the EGG trial; the YOLK trial was not different from the GLU trial. The AUC of ET-1 was only lower in EGG compared with GLU $(P<0.05)$. In addition, ET- $1_{\mathrm{AUC}}$ and glucose $\mathrm{AUC}_{\mathrm{AUC}}$ were positively correlated $(P<0 \cdot 05$; Table 2$)$. Plasma CRP, MPO and TNF- $\alpha$ and their respective AUC did not differ among trials $(P>0 \cdot 05$, online Supplementary Fig. S2). This suggests that greater NO ${ }^{\bullet}$ bioavailability by EGG and WHITE may be attributed to attenuating ET-1 independent of Ang-II and inflammation.

\section{Discussion}

Our prior study in this cohort of men with prediabetes demonstrated that replacing $25 \%$ of an OGTT ( $100 \mathrm{~g}$ ) with an isoenergetic amount of EGG or WHITE protected against PPHmediated impairments in FMD ${ }^{(27)}$. However, reducing the glucose content of the OGTT and replacing it with YOLK did not confer vasoprotection. This indicates that reducing the oral glucose load alone does not fully explain EGG- and WHITEmediated vasoprotection ${ }^{(27)}$. Indeed, the present study provides novel evidence that the previously observed vasoprotection in EGG and WHITE is mediated, in part, by attenuating dysregulated ARG metabolism and oxidative stress that otherwise decrease $\mathrm{NO}^{\bullet}$ bioavailability. Data show greater $\mathrm{NO}^{\bullet}$ metabolites in association with greater ARG and hARG and lower ADMA/ARG and SDMA/ARG. Although all egg-based meals protected against dysregulated ARG metabolism, which would be expected to improve $\mathrm{NO}^{\bullet}$ biosynthesis, $\mathrm{NO}^{\bullet}$ metabolites and FMD responses were only improved in the EGG and WHITE trials. The lack of vasoprotection observed in the YOLK trial is likely attributed to its inability to limit lipid peroxidation. Indeed, lower malondialdehyde, in association with greater $\mathrm{NO}^{\bullet}$ metabolites, occurred in the EGG and WHITE trials, but not in
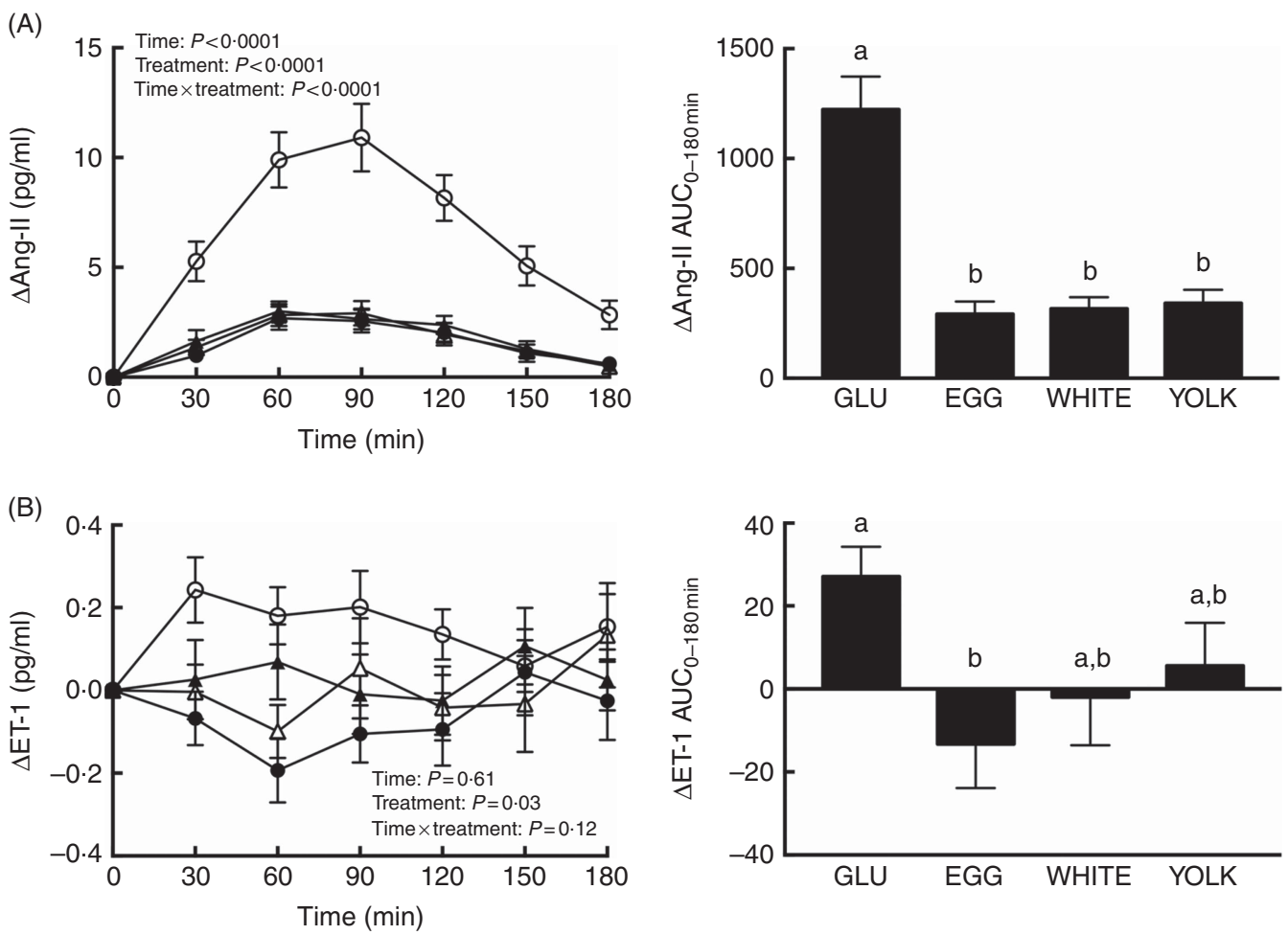

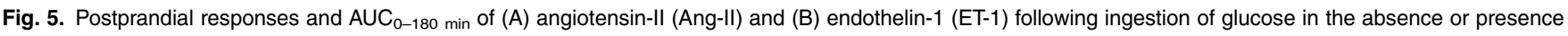
of egg-based meals by prediabetic men. Postprandial responses were analysed using two-way repeated-measures (RM) ANOVA with Bonferroni's post hoc test. $\mathrm{AUC}_{0-180}$ min was calculated using the trapezoidal rule and analysed using one-way RM ANOVA with Bonferroni's post hoc test. Values are means ( $n$ 20), with their standard errors represented by vertical bars. ${ }^{\mathrm{a}, \mathrm{b}}$ Mean values with unlike letters were significantly different $(P<0.05)$. GLU $(-\bigcirc-), 100 \mathrm{~g}$ glucose; EGG $(--)$, whole eggs; WHITE ( $\triangle$ ) , egg whites; YOLK ( $\neg$ ), egg yolks. 
the YOLK trial. Moreover, limiting increases in malondialdehyde was correlated with improvements in $\mathrm{NO}^{\circ}$ metabolites and FMD, whereas greater $\mathrm{NO}^{\bullet}$ metabolites was correlated with improved FMD responses. This suggests that vasoprotection in the EGG and WHITE trials was mediated by alleviating PPH-induced oxidative stress that otherwise impairs $\mathrm{NO}^{\circ}$-dependent vascular function. This is likely attributed to attenuating both dysregulated ARG metabolism and oxidative stress that otherwise reduce $\mathrm{NO}^{\circ}$ biosynthesis and bioavailability to impair VEF (Fig. 6).

Studies in vitro demonstrate that hydrolysates derived from WHITE exhibit free-radical scavenging activity ${ }^{(39)}$ and those from EGG lower malondialdehyde concentrations in rodents ${ }^{(29)}$. This would be expected to attenuate oxidative stress-mediated declines in $\mathrm{NO}^{\circ}$ bioavailability. In addition, peptides resulting from simulated digestion of EGG and WHITE induce ex vivo endothelium-dependent vasodilation in mesenteric arteries isolated from spontaneously hypertensive rats $^{(29,30)}$. In hyperlipidaemic adults, 6 weeks daily WHITE consumption increased fasting FMD responses ${ }^{(40)}$, which suggests improved $\mathrm{NO}^{\bullet}$ bioavailability, but this was not reported. Previously reported improvements in FMD $^{(27)}$ provided rationale for assessing $\mathrm{NO}^{\bullet}$ bioavailability in the present study. Indeed, increases in malondialdehyde that were observed in the GLU trial were attenuated in the EGG and WHITE trials in association with decreases in $\mathrm{NO}^{\circ}$ metabolites and FMD. This suggests that limiting $\mathrm{PPH}$-induced oxidative stress improves $\mathrm{NO}^{\bullet}$ bioavailability and $\mathrm{NO}^{\bullet}$-dependent vascular function. In support, $\mathrm{NO}^{\bullet}$ metabolites positively correlated with FMD, indicating greater levels of $\mathrm{NO}^{\bullet}$ metabolites were accompanied with improved vascular function. Shear stress, or the force of blood flow tangential to the endothelial surface, depending on the flow pattern, can promote $\mathrm{NO}^{\circ}$ or induce oxidative stress ${ }^{(41)}$. Thus, differences in shear stress were considered to explain EGG- and WHITE-mediated vasoprotection. However, as reported previously, shear stress did not differ among treatments ${ }^{(27)}$.

Oxidative stress is implicated in decreasing ARG availability, in part, by upregulating arginase-dependent catabolism ${ }^{(10)}$. Lower ARG availability would be expected to decrease NO${ }^{\bullet}$ biosynthesis ${ }^{(5)}$ and impair $\mathrm{VEF}^{(42)}$. In agreement, we show that increases in malondialdehyde during the GLU trial were accompanied by decreases in ARG. NO ${ }^{\bullet}$ metabolites and FMD were also correlated with ARG, suggesting that vasoprotection

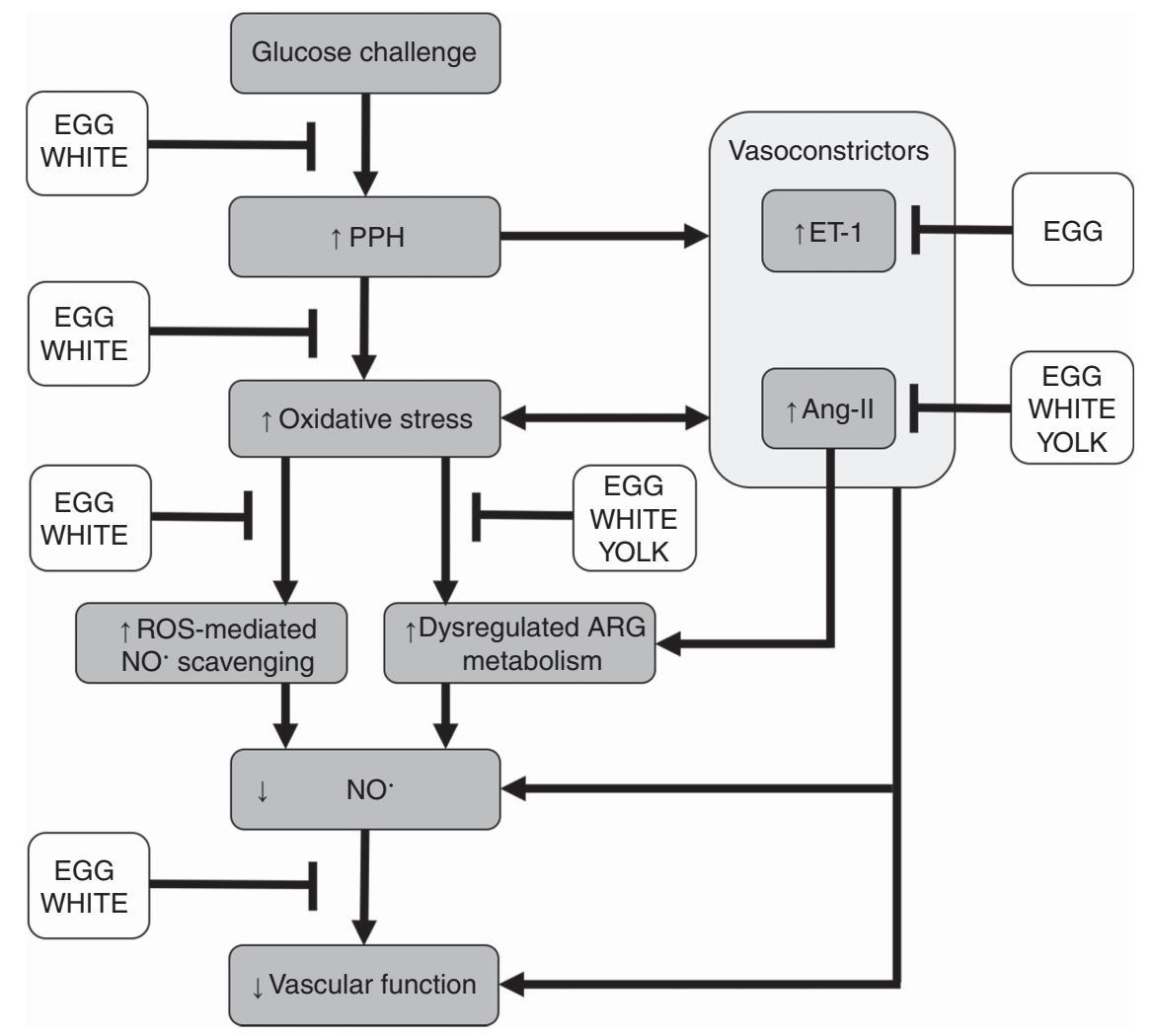

Fig. 6. Summary of the vasoprotective activities of egg-based meals on postprandial hyperglycaemia (PPH)-mediated impairments in vascular function. Ingestion of an oral glucose challenge induces PPH, which increases the vasoconstrictors angiotensin-II (Ang-II) and endothelin-1 (ET-1) while also inducing oxidative stress. Ang-II and ET-1 also contribute to oxidative stress and are upregulated by oxidative stress. Consequently, oxidative stress decreases nitric oxide bioavailability via the direct scavenging of nitric oxide by reactive oxygen species (ROS) and impairing nitric oxide biosynthesis by dysregulating arginine (ARG) metabolism. Collectively, decreases in nitric oxide impair vascular function. Co-ingestion of whole eggs (EGG) or egg whites (WHITE) with glucose attenuates PPH-mediated oxidative stress. This alleviates dysregulated ARG metabolism and scavenging of nitric oxide that otherwise reduces nitric oxide biosynthesis and bioavailability to impair vascular function. Co-ingesting egg yolks (YOLK) with glucose only attenuates dysregulated ARG metabolism but does not attenuate PPH-mediated oxidative stress, likely allowing for ROS-mediated nitric oxide scavenging and an inability to maintain vascular function. Egg-based meals differentially affect Ang-II and ET-1, which provoke dysregulated ARG metabolism, decrease nitric oxide and impair vascular function. Overall, vasoprotection mediated by whole eggs and egg whites is likely attributed to their benefits on dysregulated ARG metabolism and ROS-mediated scavenging, which improve nitric oxide bioavailability to the vasculature. 
in the EGG and WHITE trials occurred, in part, by improving ARG availability for $\mathrm{NO}^{\bullet}$ biosynthesis. This is consistent with ARG supplementation improving $\mathrm{VEF}^{(42)}$. Despite malondialdehyde not differing between the GLU and YOLK trials, and being higher than that in the EGG trial, postprandial ARG was not different between EGG and YOLK trials. This suggests that greater lipid peroxidation in YOLK compared with EGG does not fully explain ARG status. We therefore considered that circulating ARG was influenced by the dietary ARG content of test meals ${ }^{(43)}$. In support, ARG increased to the greatest extent in the WHITE trial, the treatment that also provided the greatest dietary ARG $(1.497 \mathrm{~g})^{(44)}$. However, postprandial ARG decreased and was not different between the EGG $(0.615 \mathrm{~g})$ and YOLK $(0.374 \mathrm{~g})$ trials despite an approximately $40 \%$ difference in dietary ARG. While differences in dietary ARG may help to explain increases observed in the WHITE trial, it fails to address the lack of difference between EGG and YOLK trials. Regardless, improvements in ARG status in the YOLK trial would be expected to increase $\mathrm{NO}^{\bullet}$ biosynthesis, but this was not observed. The lack of benefit of YOLK on $\mathrm{NO}^{\bullet}$ metabolites is likely attributed to its inability to limit lipid peroxidation otherwise induced by PPH. This suggests that oxidative stressmediated scavenging of $\mathrm{NO}^{\bullet}$ precluded its bioavailability to the vascular endothelium, which is consistent with the lack of improvement in vascular function in the YOLK trial ${ }^{(27)}$. In contrast, FMD responses were improved in the EGG and WHITE trials ${ }^{(27)}$, which was accompanied by greater $\mathrm{NO}^{\bullet}$ metabolites and lower lipid peroxidation. Based on these findings, future study is warranted to define the independent and additive effects of egg components to regulate ARG status and $\mathrm{NO}^{\circ}$ bioavailability in relation to $\mathrm{VEF}$.

Oxidative stress also decreases arginine:glycine amidinotransferase activity ${ }^{(14)}$, which would be expected to lower hARG to limit $\mathrm{NO}^{\bullet}$ biosynthesis ${ }^{(9)}$ and impair vascular function $^{(15)}$. In support, increases in malondialdehyde during the GLU trial were accompanied by decreases in hARG that were attenuated in the EGG and WHITE trials, but not in the YOLK trial. Furthermore, hARG was positively correlated with $\mathrm{NO}^{\bullet}$ metabolites and FMD. These findings are in agreement with evidence that higher hARG levels are associated with greater FMD responses ${ }^{(15)}$ and lower CVD risk ${ }^{(9,16)}$. However, regulation of VEF by hARG is an area of active investigation due to disparate observations ${ }^{(9,45)}$. Despite its ability to serve as an eNOS substrate and inhibit arginase activity, hARG has less binding affinity for $\operatorname{eNOS}^{(46)}$ and physiological levels (approximately $2 \mu \mathrm{m}$ ) are approximately 10-100-fold less than $\mathrm{ARG}^{(47)}$. Thus, attenuation of oxidative stress in the EGG and WHITE trials may have improved hARG status, but this may not explain the vasoprotective benefits of EGG and WHITE.

Oxidative stress also increases the accumulation of ADMA and SDMA by upregulating protein methyltransferase ${ }^{(11)}$ while preventing dimethylarginine dimethylaminohydrolasemediated catabolism of ADMA ${ }^{(6)}$. Likewise, alanine-glyoxylate aminotransferase- 2 also catabolises ADMA and SDMA ${ }^{(48)}$ but the role of oxidative stress on alanine-glyoxylate aminotransferase- 2 activity has not been reported. Oxidative stress decreases the activity of cationic amino acid transporter to limit cellular clearance of ADMA and SDMA ${ }^{(48,49)}$. Collectively, this decreases $\mathrm{NO}^{\bullet}$ biosynthesis $^{(5)}$, consistent with ADMA and SDMA competitively inhibiting cellular uptake of $\mathrm{ARG}^{(12,13)}$ and ADMA competitively inhibiting eNOS ${ }^{(11)}$. In agreement, we show that increases in malondialdehyde during the GLU trial were accompanied by increases in ADMA/ARG and SDMA/ ARG that were similarly attenuated by EGG and WHITE. In addition, ADMA/ARG and SDMA/ARG negatively correlated with $\mathrm{NO}^{\bullet}$ metabolites and FMD, suggesting that PPH-mediated oxidative stress limited $\mathrm{NO}^{\bullet}$ biosynthesis to impair vascular function by inhibiting cellular ARG uptake and eNOS activity. Our findings are consistent with those demonstrating that $\mathrm{PPH}$ impaired VEF, in part, by increasing ADMA/ARG following an OGTT $^{(4)}$. They are also consistent with evidence in vitro showing that SDMA dose-dependently increases oxidative stress and reduces $\mathrm{NO}^{\bullet}$ metabolites otherwise abrogated by $\mathrm{ARG}^{(12)}$. This would be expected to impair VEF and is supported by evidence associating greater SDMA with increased CVD risk in diabetic subjects ${ }^{(50)}$. Interestingly, compared with the GLU trial, increases in ADMA/ARG and SDMA/ARG were attenuated in the YOLK trial despite no improvements in malondialdehyde. However, this did not improve $\mathrm{NO}^{\bullet}$ metabolites or FMD responses and suggests that improving $\mathrm{NO}^{\bullet}$ biosynthesis alone does not fully confer vasoprotection. Rather, decreasing oxidative stress-mediated scavenging of $\mathrm{NO}^{\bullet}$ is also needed to facilitate $\mathrm{NO}^{*}$ bioavailability and maintain VEF (Fig. 6). Improvements in ADMA/ARG and SDMA/ARG suggest bioactive egg components may inhibit protein methyltransferase or increase activities of dimethylarginine dimethylaminohydrolase, alanine-glyoxylate aminotransferase-2 and/or cationic amino acid transporter but requires future investigation.

Apart from dysregulating ARG metabolism, PPH-mediated oxidative stress also impairs vascular function by oxidising the eNOS cofactor $\mathrm{BH}_{4}^{(18,51)}$ and by increasing Ang-II ${ }^{(20,52)}$ and ET-1 ${ }^{(21,53)}$ to decrease eNOS expression and activity ${ }^{(22,23)}$. Thus, attenuation of oxidative stress in the EGG and WHITE trials, but not the YOLK trials, may have conferred vasoprotection through these pathways. However, $\mathrm{BH}_{4} / \mathrm{BH}_{2}$ was unaffected by treatment, suggesting increased oxidative stress was independent of eNOS uncoupling ${ }^{(17)}$. This may be due to participants having adequate vitamin $\mathrm{C}$ concentrations (approximately $40 \mu \mathrm{M})^{(54)}$ to recycle $\mathrm{BH}_{4}^{(55)}$. All egg-based meals protected against increases in Ang-II, which may be due to egg-derived peptides inhibiting angiotensin-converting enzyme ${ }^{(39)}$. In bovine aortic endothelial cells, Ang-II upregulates arginase to decrease $\mathrm{NO}^{\bullet}$ generation ${ }^{(56)}$. In addition, incubation of rat vascular smooth muscle cells with Ang-II increased protein methyltransferase and decreased dimethylarginine dimethylaminohydrolase and cationic amino acid transporter activity leading to increased ADMA levels. Thus, protection against increases in Ang-II by egg-based meals may have limited AngII-mediated decreases in ARG and increases in ADMA and SDMA (Fig. 6), but this requires future study. Lastly, despite evidence showing that $\mathrm{PPH}$ increases pro-inflammatory mediators $^{(24)}$ and egg ingestion (2-3 eggs/d for 4 weeks) improves antioxidant status ${ }^{(57)}$, these responses were unaffected in the present study. Thus, vasoprotection in the EGG and WHITE trials, at least postprandially, occurred independent of lowering pro-inflammatory mediators or improving antioxidant status. 
Oxidative stress mediated by acute hyperglycaemia plays a central role in reducing $\mathrm{NO}^{\circ}$ bioavailability and impairing $\mathrm{VEF}^{(58)}$. Co-ingestion of EGG or WHITE, but not YOLK, with an OGTT attenuates increases in glycaemia and decreases in FMD responses ${ }^{(27)}$. The observed vasoprotection was mediated, in part, at the level of the gut by increasing cholecystokinin ${ }^{(27)}$, which functions to delay gastric emptying ${ }^{(59)}$. This supports a pre-absorptive mechanism to attenuate glycaemia and downstream oxidative stress. Together with the present study, the available evidence suggests a link between gut-level responses and vascular health. Hyperglycaemia induces oxidative stress through multiple mechanisms including mitochondrial superoxide production, proinflammatory mediators, advanced glycation end products, eNOS uncoupling and increased enzymatic (i.e. NADPH oxidase and protein kinase C) activity $^{(6,60)}$. However, evidence in vitro indicates that hyperglycaemia-mediated activation of these pathways is ameliorated by blocking glucose-induced mitochondrial superoxide production ${ }^{(61)}$. Furthermore, mitochondrial-derived reactive oxygen species activates NADPH oxidase, which in turn, stimulates mitochondrial reactive oxygen species generation, thereby creating a detrimental cycle of oxidative stress ${ }^{(62)}$. Thus, vasoprotection in the EGG and WHITE trials, but not the YOLK trial, may be potentially attributed to limiting glucoseinduced mitochondrial reactive oxygen species production and/or NADPH oxidase activation, which requires further study. In addition, increased malondialdehyde in the YOLK trial may be, in part, due to oxidation of egg yolk lipids during digestion $^{(63)}$, which could contribute to $\mathrm{NO}^{\bullet}$ scavenging and limit its bioavailability for improving VEF. However, increases in malondialdehyde were attenuated in the EGG trial, which may be attributed to WHITE limiting egg yolk lipid oxidation ${ }^{(63)}$. Ultimately, limiting reactive oxygen species (e.g. superoxide), which reacts with $\mathrm{NO}^{\circ}$, would be expected to increase $\mathrm{NO}^{\circ}$ bioavailability to improve VEF.

Our findings show that PPH and lipid peroxidation were attenuated, and vascular function improved in the EGG and WHITE trials only. In addition, there were no statistically different outcomes between the EGG and WHITE trials, suggesting that the WHITE fraction of EGG is functionally responsible for vasoprotection observed previously ${ }^{(27)}$. In support, peptides resulting from enzymatic digestion of WHITE proteins (e.g. ovalbumin, ovotransferrin, ovomucin and lysozyme) exhibit glycaemia-lowering, antioxidant, and $\mathrm{NO}^{\circ}$-producing activities ${ }^{(39)}$. Thus, in addition to cholecystokinin-mediated delays in gastric emptying, bioactive peptides resulting from digestion may mediate a postabsorptive vasoprotective mechanism. However, these peptides and their circulating levels have not been studied in humans. In addition, future postprandial studies should examine other protein-rich foods (e.g. milk) to determine if vasoprotection is unique to eggs. The present study was designed to deliver isoenergetic meals reflective of typical breakfast intakes by American men ${ }^{(27)}$, and future studies should consider potential dose-dependent effects of eggs and how modifying meal composition with protein and fat regulates postprandial responses. Our studies were specifically limited to a 180-min postprandial period consistent with glucose ingestion transiently impairing vascular function ${ }^{(4,27)}$. However, some of the biomarkers reported herein (i.e. ARG and metabolites) did not return to baseline levels by $180 \mathrm{~min}$. Thus, future studies of longer duration are needed to evaluate the effects of subsequent meals on postprandial health. Lastly, chronic egg consumption attenuates PPH that is induced by an OGTT $^{(64)}$. It also decreases fasting malondialdehyde ${ }^{(29)}$ and increases FMD responses ${ }^{(40)}$. Thus, chronic studies are needed to examine egg consumption on postprandial glycaemia and oxidative stress in relation to VEF. However, a benefit of postprandial studies is that they permit the direct evaluation of food and its components that is often confounded by the displacement of other foods during chronic studies.

In conclusion, the present study demonstrates that coingesting EGG or WHITE, but not YOLK, with an oral glucose challenge protects against postprandial impairments in vascular function by limiting $\mathrm{PPH}$-induced oxidative stress that otherwise scavenges $\mathrm{NO}^{\bullet}$ to decrease its bioavailability (Fig. 6). Thus, replacing carbohydrates in a meal for EGG or WHITE may be a beneficial dietary strategy to attenuate PPH-mediated oxidative stress that otherwise decreases $\mathrm{NO}^{\bullet}$ bioavailability to impair vascular function and increase CVD risk. Consistent with PPH being a better predictor of long-term CVD risk compared with fasting glucose ${ }^{(26)}$, a need exists to validate dietary strategies that limit meal-induced injuries to the vascular endothelium that are attributed to PPH and its downstream oxidative stress responses. In the absence of effective approaches, the accumulation of postprandial insults is expected to increase CVD risk, which remains the leading cause of mortality in the $\mathrm{USA}^{(1)}$.

\section{Acknowledgements}

The authors thank Meredith Moller and John Bouranis for their assistance with biochemical analyses.

The present study was funded by the American Egg Board/ Egg Nutrition Center. American Egg Board/Egg Nutrition Center had no role in the design, analysis or writing of this article.

The authors' contributions are as follows: R. S. B., J. S. V. and E. M. were responsible for the study design; J. D. M., C. C., E. J. R., J. L. and F. A. V. were responsible for collecting and analysing data; J. D. M. and R. S. B. wrote the initial draft of the manuscript and all authors contributed to the editing and review of this manuscript. All authors read and approved the final manuscript.

The authors declare that there are no conflicts of interest.

\section{Supplementary material}

For supplementary material/s referred to in this article, please visit https://doi.org/10.1017/S0007114518002192

\section{References}

1. Mozaffarian D, Benjamin EJ, Go AS, et al. (2016) Heart disease and stroke statistics-2016 update: a report from the American Heart Association. Circulation 133, e38-e360.

2. Loader J, Montero D, Lorenzen C, et al. (2015) Acute hyperglycemia impairs vascular function in healthy and 
cardiometabolic diseased subjects: systematic review and meta-analysis. Arterioscler Thromb Vasc Biol 35, 2060-2072.

3. Versari D, Daghini E, Virdis A, et al. (2009) Endothelial dysfunction as a target for prevention of cardiovascular disease. Diabetes Care 32, S314-S321.

4. Mah E, Noh SK, Ballard KD, et al. (2011) Postprandial hyperglycemia impairs vascular endothelial function in healthy men by inducing lipid peroxidation and increasing asymmetric dimethylarginine:arginine. J Nutr 141, 1961-1968.

5. Naseem KM (2005) The role of nitric oxide in cardiovascular diseases. Mol Aspects Med 26, 33-65.

6. Mah E \& Bruno RS (2012) Postprandial hyperglycemia on vascular endothelial function: mechanisms and consequences. Nutr Res 32, 727-740.

7. Harris RA, Nishiyama SK, Wray DW, et al. (2010) Ultrasound assessment of flow-mediated dilation. Hypertension $\mathbf{5 5}$, 1075-1085.

8. Matsuzawa Y, Kwon TG, Lennon RJ, et al. (2015) Prognostic value of flow-mediated vasodilation in brachial artery and fingertip artery for cardiovascular events: a systematic review and meta-analysis. J Am Heart Assoc 4, e002270-e002284.

9. Papageorgiou N, Androulakis E, Papaioannou S, et al. (2015) Homoarginine in the shadow of asymmetric dimethylarginine: from nitric oxide to cardiovascular disease. Amino Acids 47, 1741-1750.

10. Ryoo S, Lemmon CA, Soucy KG, et al. (2006) Oxidized lowdensity lipoprotein-dependent endothelial arginase II activation contributes to impaired nitric oxide signaling. Circ Res 99 , 951-960.

11. Boger RH, Sydow K, Borlak J, et al. (2000) LDL cholesterol upregulates synthesis of asymmetrical dimethylarginine in human endothelial cells: involvement of $S$-adenosylmethionine-dependent methyltransferases. Circ Res 87, 99-105.

12. Bode-Boger SM, Scalera F, Kielstein JT, et al. (2006) Symmetrical dimethylarginine: a new combined parameter for renal function and extent of coronary artery disease. J Am Soc Nephrol 17, 1128-1134.

13. Leiper J \& Vallance P (1999) Biological significance of endogenous methylarginines that inhibit nitric oxide synthases. Cardiovasc Res $\mathbf{4 3}, 542-548$

14. Ito T, Yufu K, Mori A, et al. (1996) Oxidative stress alters arginine metabolism in rat brain: effect of sub-convulsive hyperbaric oxygen exposure. Neurochem Int 29, 187-195.

15. Valtonen P, Laitinen T, Lyyra-Laitinen T, et al. (2008) Serum L-homoarginine concentration is elevated during normal pregnancy and is related to flow-mediated vasodilatation. Circ J 72, 1879-1884.

16. Atzler D, Schwedhelm E \& Choe CU (2015) L-Homoarginine and cardiovascular disease. Curr Opin Clin Nutr Metab Care 18, 83-88.

17. Vasquez-Vivar J, Kalyanaraman B, Martasek P, et al. (1998) Superoxide generation by endothelial nitric oxide synthase: the influence of cofactors. Proc Natl Acad Sci USA 95 , 9220-9225.

18. Crabtree MJ, Smith CL, Lam G, et al. (2008) Ratio of 5,6,7,8tetrahydrobiopterin to 7,8-dihydrobiopterin in endothelial cells determines glucose-elicited changes in NO vs. superoxide production by eNOS. Am J Physiol Heart Circ Physiol 294, H1530-H1540.

19. Takeda M, Yamashita T, Shinohara M, et al. (2009) Plasma tetrahydrobiopterin/dihydrobiopterin ratio: a possible marker of endothelial dysfunction. Circ J 73, 955-962.

20. Day RT, de Cássia Cavaglieri R, Tabatabaimir H, et al. (2010) Acute hyperglycemia rapidly stimulates VEGF mRNA translation in the kidney. Role of angiotensin type 2 receptor (AT2). Cell Signal 22, 1849-1857.
21. Grassi D, Desideri G, Necozione S, et al. (2012) Protective effects of flavanol-rich dark chocolate on endothelial function and wave reflection during acute hyperglycemia. Hypertension $\mathbf{6 0}, 827-832$.

22. Loot AE, Schreiber JG, Fisslthaler B, et al. (2009) Angiotensin II impairs endothelial function via tyrosine phosphorylation of the endothelial nitric oxide synthase. I Exp Med 206, 2889-2896.

23. Ramzy D, Rao V, Tumiati LC, et al. (2006) Elevated endothelin-1 levels impair nitric oxide homeostasis through a PKC-dependent pathway. Circulation 114, I319-I326.

24. Esposito K, Nappo F, Marfella R, et al. (2002) Inflammatory cytokine concentrations are acutely increased by hyperglycemia in humans: role of oxidative stress. Circulation $\mathbf{1 0 6}$, 2067-2072.

25. Siti HN, Kamisah Y \& Kamsiah J (2015) The role of oxidative stress, antioxidants and vascular inflammation in cardiovascular disease (a review). Vascul Pharmacol 71, 40-56.

26. Decode Study Group, The European Diabetes Epidemiology Group (2001) Glucose tolerance and cardiovascular mortality: comparison of fasting and 2-hour diagnostic criteria. Arch Intern Med 161, 397-405.

27. McDonald JD, Chitchumroonchokchai C, Li J, et al. (2018) Replacing carbohydrate during a glucose challenge with the egg white portion or whole eggs protects against postprandial impairments in vascular endothelial function in prediabetic men by limiting increases in glycaemia and lipid peroxidation. Br J Nutr 119, 259-270.

28. Davalos A, Miguel M, Bartolome B, et al. (2004) Antioxidant activity of peptides derived from egg white proteins by enzymatic hydrolysis. J Food Prot 67, 1939-1944.

29. Jahandideh F, Majumder K, Chakrabarti S, et al. (2014) Beneficial effects of simulated gastro-intestinal digests of fried egg and its fractions on blood pressure, plasma lipids and oxidative stress in spontaneously hypertensive rats. PLOS ONE $\mathbf{9}$, e115006.

30. Matoba N, Usui $\mathrm{H}$, Fujita $\mathrm{H}$, et al. (1999) A novel antihypertensive peptide derived from ovalbumin induces nitric oxide-mediated vasorelaxation in an isolated SHR mesenteric artery. FEBS Lett 452, 181-184.

31. American Diabetes Association (2011) Standards of medical care in diabetes - 2011. Diabetes Care 34, S11-S61.

32. U.S. Department of Agriculture, Agricultural Research Service (2016) Nutrient Intakes from Food and Beverages: Mean Amounts Consumed per Individual, by Gender and Age, What We Eat in America, NHANES 2013-2014. Beltsville, MD: U.S. Department of Agriculture, Agricultural Research Service.

33. Bruno RS, Dugan CE, Smyth JA, et al. (2008) Green tea extract protects leptin-deficient, spontaneously obese mice from hepatic steatosis and injury. J Nutr 138, 323-331.

34. Gleize B, Steib M, André M, et al. (2012) Simple and fast HPLC method for simultaneous determination of retinol, tocopherols, coenzyme $\mathrm{Q}(10)$ and carotenoids in complex samples. Food Chem 134, 2560-2564.

35. Valdes C, Arauna D, Gonzalez D, et al. (2017) Simplified HPLC methodology for quantifying biological pterins by selective oxidation. J Chromatogr B Analyt Technol Biomed Life Sci 1055-1056, 113-118.

36. Biondi R, Ambrosio G, De Pascali F, et al. (2012) HPLC analysis of tetrahydrobiopterin and its pteridine derivatives using sequential electrochemical and fluorimetric detection: application to tetrahydrobiopterin autoxidation and chemical oxidation. Arch Biochem Biophys 520, 7-16.

37. Bland JM \& Altman DG (1995) Calculating correlation coefficients with repeated observations: Part 1-correlation within subjects. BMJ 310, 446. 
38. Gross M, Yu X, Hannan P, et al. (2003) Lipid standardization of serum fat-soluble antioxidant concentrations: the YALTA study. Am J Clin Nutr 77, 458-466.

39. Liu YF, Oey I, Bremer P, et al. (2017) Bioactive peptides derived from egg proteins: a review. Crit Rev Food Sci Nutr (Epublication ahead of print version 22 August 2017).

40. Njike V, Faridi Z, Dutta S, et al. (2010) Daily egg consumption in hyperlipidemic adults-effects on endothelial function and cardiovascular risk. Nutr $5 \mathbf{9}, 28$.

41. Chatzizisis YS, Coskun AU, Jonas M, et al. (2007) Role of endothelial shear stress in the natural history of coronary atherosclerosis and vascular remodeling: molecular, cellular, and vascular behavior. J Am Coll Cardiol 49, 2379-2393.

42. Preli RB, Klein KP \& Herrington DM (2002) Vascular effects of dietary L-arginine supplementation. Atherosclerosis 162, 1-15.

43. Mariotti Fo, Huneau JFo, Szezepanski I, et al. (2007) Meal amino acids with varied levels of arginine do not affect postprandial vascular endothelial function in healthy young men. J Nutr 137, 1383-1389.

44. U.S. Department of Agriculture, Agricultural Research Service (2016) USDA national nutrient database for standard reference, release 28 . Nutrient data laboratory home page. https:// ndb.nal.usda.gov/ndb/ (accessed May 2018).

45. Atzler D, Schonhoff M, Cordts K, et al. (2016) Oral supplementation with L-homoarginine in young volunteers. Br J Clin Pharmacol 82, 1477-1485.

46. Moali C, Boucher JL, Sari MA, et al. (1998) Substrate specificity of NO synthases: detailed comparison of L-arginine, homo-Larginine, their $\mathrm{N}$-omega-hydroxy derivatives, and $\mathrm{N}$-omegahydroxynor-L-arginine. Biochemistry 37, 10453-10460.

47. Michel T (2013) R is for arginine: metabolism of arginine takes off again, in new directions. Circulation 128, 1400-1404.

48. Tain YL \& Hsu CN (2017) Toxic dimethylarginines: asymmetric dimethylarginine (ADMA) and symmetric dimethylarginine (SDMA). Toxins (Basel) 9, E92.

49. Luo Z, Teerlink T, Griendling K, et al. (2010) Angiotensin II and NADPH oxidase increase ADMA in vascular smooth muscle cells. Hypertension 56, 498-504.

50. Zobel EH, von Scholten BJ, Reinhard H, et al. (2017) Symmetric and asymmetric dimethylarginine as risk markers of cardiovascular disease, all-cause mortality and deterioration in kidney function in persons with type 2 diabetes and microalbuminuria. Cardiovasc Diabetol 16, 88.

51. Ihlemann N, Rask-Madsen C, Perner A, et al. (2003) Tetrahydrobiopterin restores endothelial dysfunction induced by an oral glucose challenge in healthy subjects. Am J Physiol Heart Circ Physiol 285, H875-H882.

52. Keidar S, Heinrich R, Kaplan M, et al. (2002) Oxidative stress increases the expression of the angiotensin-II receptor type 1 in mouse peritoneal macrophages. J Renin Angiotensin Aldosterone Syst 3, 24-30.

53. Ruef J, Moser M, Kubler W, et al. (2001) Induction of endothelin-1 expression by oxidative stress in vascular smooth muscle cells. Cardiovasc Pathol 10, 311-315.

54. Schleicher RL, Carroll MD, Ford ES, et al. (2009) Serum vitamin C and the prevalence of vitamin $\mathrm{C}$ deficiency in the United States: 2003-2004 National Health and Nutrition Examination Survey (NHANES). Am J Clin Nutr 90, 1252-1263.

55. Kuzkaya N, Weissmann N, Harrison DG, et al. (2005) Interactions of peroxynitrite with uric acid in the presence of ascorbate and thiols: implications for uncoupling endothelial nitric oxide synthase. Biochem Pharmacol 70, 343-354.

56. Shatanawi A, Lemtalsi T, Yao L, et al. (2015) Angiotensin II limits NO production by upregulating arginase through a p38 MAPK-ATF-2 pathway. Eur J Pharmacol 746, 106-114.

57. DiMarco DM, Norris GH, Millar CL, et al. (2017) Intake of up to 3 eggs per day is associated with changes in HDL function and increased plasma antioxidants in healthy, young adults. J Nutr 147, 323-329.

58. Cai H \& Harrison DG (2000) Endothelial dysfunction in cardiovascular diseases: the role of oxidant stress. Circ Res 87, 840-844.

59. Liddle RA, Morita ET, Conrad CK, et al. (1986) Regulation of gastric emptying in humans by cholecystokinin. J Clin Invest 77, 992-996.

60. Brownlee M (2001) Biochemistry and molecular cell biology of diabetic complications. Nature 414, 813-820.

61. Nishikawa T, Edelstein D, Du XL, et al. (2000) Normalizing mitochondrial superoxide production blocks three pathways of hyperglycaemic damage. Nature 404, 787-790.

62. Daiber A, Di Lisa F, Oelze M, et al. (2017) Crosstalk of mitochondria with NADPH oxidase via reactive oxygen and nitrogen species signalling and its role for vascular function. Br J Pharmacol 174, 1670-1689.

63. Kobayashi H, Sasahara R, Yoda S, et al. (2017) Egg white hydrolysate inhibits oxidation in mayonnaise and a model system. Biosci Biotechnol Biochem 81, 1206-1215.

64. Pearce KL, Clifton PM \& Noakes M (2011) Egg consumption as part of an energy-restricted high-protein diet improves blood lipid and blood glucose profiles in individuals with type 2 diabetes. Br J Nutr 105, 584-592. 